\title{
Acute Generalized Exanthematous Pustulosis Caused by Daptomycin
}

\author{
Teoh Yee Leng, M.D., Mark Koh Jean Aan, M.D., Michelle Chan, M.D. ${ }^{1}$, Liu Tsun Tsien, M.D. \\ Department of Dermatology, Changi General Hospital, Singapore 529889, ${ }^{1}$ Department of Pathology, Singapore General Hospital, \\ Singapore 169608, Singapore
}

Daptomycin, a lipopeptide antibiotic with similar action as vancomycin, is used to treat complicated skin and soft tissue infections caused by resistant Gram-positive bacteria, including methicillin-resistant Staphylococcus aureus, penicillin-resistant streptococci, and vancomycin-resistant enterococci. Acute generalized exanthematous pustulosis (AG$\mathrm{EP})$, characterized by acute onset of numerous sterile, nonfollicular pinhead sized pustules, is common secondary to drugs, in particular, antibiotics. We present the first case of AGEP following the use of daptomycin. (Ann Dermatol 23(S3) S288 S289, 2011)

\section{-Keywords-}

Acute generalized exanthematous pustulosis, Daptomycin

\section{INTRODUCTION}

Acute generalized exanthematous pustulosis (AGEP), a self-limiting drug eruption, commonly occur secondary to antibiotics. We present a first case of AGEP after treatment with daptomycin, an antibiotic used to treat infections caused by methicillin-resistant Staphylococcus aureus, penicillin-resistant streptococci and vancomycin-resistant enterococci.

Received July 12, 2010, Revised November 1, 2010, Accepted for publication November 1, 2010

Corresponding author: Mark Koh Jean Aan, M.D., Department of Dermatology, Changi General Hospital, Singapore 529889, Singapore. Tel: 65-9689-2431, Fax: 65-6788-0933, E-mail: docmark@ pacific.net.sg

This is an Open Access article distributed under the terms of the Creative Commons Attribution Non-Commercial License (http:// creativecommons.org/licenses/by-nc/3.0) which permits unrestricted non-commercial use, distribution, and reproduction in any medium, provided the original work is properly cited.

\section{CASE REPORT}

A 57-year-old man, with underlying problems of hypertension and poorly controlled diabetes mellitus, was admitted for right foot osteomyelitis with septic shock. He had no history of skin disease. He underwent a right below-knee amputation and was post-operatively started on vancomycin for methicillin-resistant Staphylococcus aureus (MRSA) bacteraemia. However, due to poor clinical response and persistently high vancomycin minimum inhibitory concentration levels, vancomycin was switched to intravenous daptomycin.

On day 24 of daptomycin administration, the patient developed a non-pruritic generalised maculopapular erythematous rash over the trunk and limbs, with no mucosal involvement. He had a temperature of $38.6^{\circ} \mathrm{C}$, leucocytosis of $17.6 \times 10^{9} / \mathrm{L}$, and an absolute neutrophil count

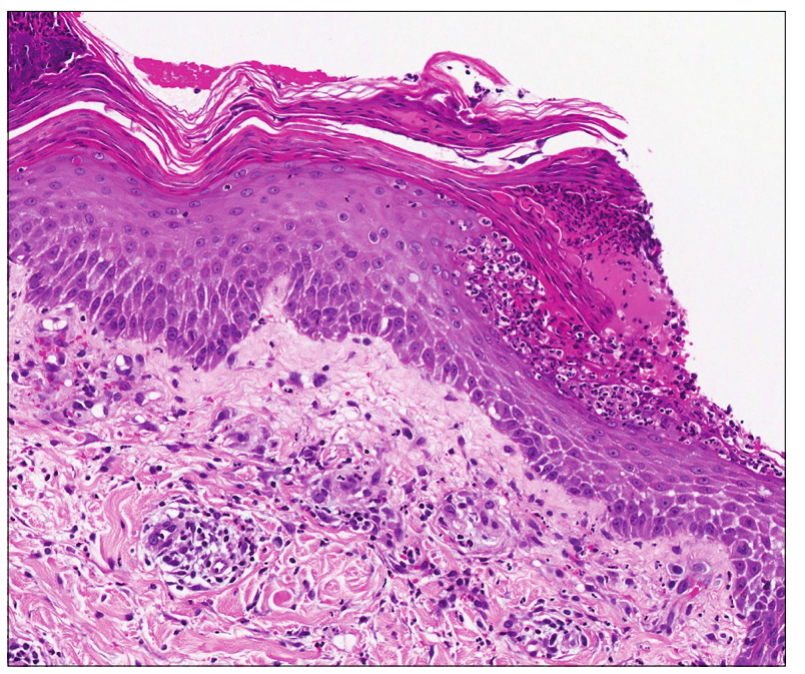

Fig. 1. Histology shows subcorneal pustules with perivascular lymphoplasmacytic infiltrates admixed with neutrophils and occasional eosinophils $(\mathrm{H} \& \mathrm{E}, \times 100)$. 
of $8.4 \times 10^{3} / \mu \mathrm{l}$. Skin scraping for fungal microscopy was negative. Six days later, the rash became more extensive with small pit-point pustules and superficial scaling. A skin biopsy (Fig. 1) showed subcorneal pustules with a perivascular infiltrate consisting mainly of lymphocytes and plasma cells with some neutrophils and scattered eosinophils. No fungal hyphae or spores were seen.

A diagnosis of acute generalized exanthematous pustulosis (AGEP) due to daptomycin was made after excluding other possible causative drugs. However, in view of the MRSA bacteraemia and recurrent osteomyelitis, a decision was made to complete 6 weeks of daptomycin therapy. He was treated symptomatically with topical corticosteroids, moisturisers, and antihistamines. Two weeks after completing the daptomycin course, the rash improved, with residual scattered superficial scaly patches and postinflammatory hyperpigmentation.

\section{DISCUSSION}

AGEP is an eruption triggered most often by drugs, and antibiotics (penicillins, quinolones, and macrolides) are the most frequent cause. Daptomycin, a lipopeptide antibiotic with similar action as vancomycin, is used to treat complicated skin and soft tissue infections caused by resistant Gram-positive bacteria including $\mathrm{MRSA}^{1}$, penicillin-resistant streptococci and vancomycin-resistant enterococci ${ }^{2}$. Other causes include viral infections e.g. enterovirus, parvovirus B19, adenovirus, cytomegalovirus, Epstein-Barr virus and hepatitis B virus, as well as hypersensitivity reactions to mercury ${ }^{3,4}$. The median duration of treatment before onset of the rash is $1 \sim 3$ weeks.

Histological findings include spongiform sub-corneal pustules with perivascular infiltrates of neutrophils and some eosinophils. The diagnosis of AGEP is made based on a clinical-pathological correlation, as there are no other definitive diagnostic markers. Response to discontinuation of medication supports the diagnosis. Differential diagnoses include pustular psoriasis, anticonvulsive hypersensitivity syndrome, subcorneal pustular dermatosis and superficial fungal infections, Sweet's syndrome, toxic epidermal necrolysis and erythema multiforme, particularly if target lesions, skin sloughing or involvement of mucous membranes are present ${ }^{5}$.

AGEP resolves upon discontinuation of the implicated medication, which may be the only required therapeutic measure. Some patients may require topical corticosteroids to help alleviate symptoms.

Our patient had clinical and histological findings consistent with AGEP. In view of the timing of onset and resolution of the rash with regards to the course of daptomycin, and after exclusion of other causative drugs, our patient most likely had AGEP secondary to daptomycin. To the best of our knowledge, this is the first report of AGEP caused by daptomycin.

\section{REFERENCES}

1. Carpenter CF, Chambers HF. Daptomycin: another novel agent for treating infections due to drug-resistant grampositive pathogens. Clin Infect Dis 2004;38:994-1000.

2. Streit JM, Jones RN, Sader HS. Daptomycin activity and spectrum: a worldwide sample of 6737 clinical Grampositive organisms. J Antimicrob Chemother 2004;53:669674.

3. Lerch M, Bircher AJ. Systemically induced allergic exanthem from mercury. Contact Dermatitis 2004;50:349-353.

4. Haro-Gabaldón V, Sánchez-Sánchez-Vizcaino J, Ruiz-Avila P, Gutiérrez-Fernández J, Linares J, Naranjo-Sintes R. Acute generalized exanthematous pustulosis with cytomegalovirus infection. Int J Dermatol 1996;35:735-737.

5. Sidoroff A, Dunant A, Viboud C, Halevy S, Bavinck JN, Naldi L, et al. Risk factors for acute generalized exanthematous pustulosis (AGEP)-results of a multinational casecontrol study (EuroSCAR). Br J Dermatol 2007;157:989-996. 